\author{
Başak Karakurum \\ Seniha Naldöken \\ Hülya Ortapamuk \\ Serap Üçler \\ Levent E. Inan
}

\section{Menstrual migraine and SPECT}

\section{Received: 20 March 2002}

Accepted in revised form: 2 October 2002

B. Karakurum $(凹) \cdot S$. Uçler • L.E. Inan Department of Neurology,

Ministry of Health Ankara Research and Training Hospital, Başkent University, Ankara, Turkey

e-mail: bkarakurum@hotmail.com

Tel.: +90-322-454-7613

Fax: +90-322-327-1273

S. Naldoken $\bullet$ H. Ortapamuk

Department of Nuclear Medicine,

Faculty of Medicine,

Ministry of Health Ankara Research and

Training Hospital,

Başkent University,

Ankara, Turkey

\begin{abstract}
Menstrual migraine (MM) pathogenesis has not yet been clarified, even though several hypotheses have been formulated. In the present research, we investigated cerebral blood flow in 15 patients with MM. Brain SPECT was performed in the middle of the menstrual cycle and during an MM attack. Although some modifications in cerebral blood flow were observed in both periods, no statistically significant differences were found.
\end{abstract}

Key words Menstruation $•$ Migraine $•$ SPECT - Cerebral blood flow •

Menstrual migraine

\section{Introduction}

Migraine headaches are common, especially in reproductive life [1]. Although there is no sex difference in the prevalence of migraine in prepubertal age, it occurs more frequently in adult women than in men in post-pubertal age [1]. Migraine attacks are linked to the period of menses in the second decade of life; the condition improves during the second and third trimesters of pregnancy [2-5]. Hence, most of the authors think that hormonal factors may play a role in patients with migraine [1-5]. However, the pathophysiology of menstrual migraine (MM) has not yet been clearly defined, and MM has not been categorised in the International Headache Society (IHS) classification [1].

Some recent studies investigated the relationship between migraine with aura (MWA) and menstrual migraine. In one study, no difference in the occurrence of pure MM emerged between patients with or without aura [6]. Prevalence of MM has been reported to be 7\%-70\% [7]. The lack of a standard definition for MM explains this discrepancy. After the definition of MM by MacGregor et al. [1], the diagnosis of MM attack became easier. MacGregor et al. [1] defined MM as attacks that occurred regularly on or between days -2 to +3 of the menstrual cycle and at no other time.

We performed single photon emission computed tomography (SPECT) of the brain in patients with true MM during the migraine attack and in the midcycle to investigate possible changes in cerebral blood flow.

\section{Patients and methods}

This study was carried out between September 1998 and February 2000 in the headache outpatient clinic of the Neurology 
Department of Ankara Research and Training Hospital. We considered 30 female patients who were diagnosed with migraine according to the diagnostic criteria of the International Headache Society (IHS) published in 1988 [5].

The 30 patients were asked to keep a headache daily which consisted of questions about the days with headache, localisation, laterality, intensity of pain, presence of nausea, vomiting, photophobia and phonophobia, and whether the pain increased with walking or not. The intensity of pain was scored on a visual analog scale (VAS) in which 0 represented no pain and 10 meant the worst pain imaginable. Menstrual cycles of the patients were regular. None of the patients used oral contraceptives.

After evaluation of the patients with their dairies, 15 patients whose headache met the definition of MM by MacGregor et al. [1] were included in the study. These patients were followed for two cycles of menstrual periods. The patients were informed about the study and none of the patients used antimigraine treatments during the study period. We noticed their menstruation and migraine attacks.

Fourteen days before the next expected menstruation period was accepted as probable ovulation time. Thus, brain SPECT was performed at this time (midcycle), which was chosen individually for each patient. The patients did not have headache in probable ovulation time. Brain SPECT was performed during the MM attack. The patients phoned us when they had MM attack and went to the hospital for SPECT analysis.

Brain SPECT was performed with rotating gamma camera system. Five minutes before SPECT, $15 \mathrm{mCi}$ Tc 99 HMPAO was injected intravenously with the patient lying supine with eyes closed. Data were acquired in a $64 \times 64$ matrix through $360^{\circ}$ rotation at intervals for 15 seconds per view.

Two experienced nuclear medicine physicians, who had no clinical information about the patients, did visual interpretation of the cortical uptake. Attention was given to the presence of any brain perfusion abnormality. Quantitative evaluation of the cortical uptake was evaluated with Ceretec pocket programme. Regions of interest were drawn on the images to generate mean counts per pixel, and normalized to the cerebellum. Perfusion activities in the frontal, temporal, parietal, and occipital cortices were compared between midcycle and MM attack.

Wilcoxon's matched pairs (signed ranks) and ANOVA tests were employed for statistical analysis. A $p$ value $<0.05$ was accepted to be statistically significant.

\section{Results}

The mean age of the 15 patients with menstrual migraine (MM) was 37.8 years (SD, 8.0 years). Mean MM migraine duration was 13.0 years (SD, 1.2 years) and mean pain duration was 2.5 days (SD, 0.7 days). The intensity of pain, as scored on VAS, was $7.9 \pm 1.5$ (mean \pm SD). MM attacks generally occurred on day 1 or 2 of the menstrual cycle $(1.0 \pm 0.5$, mean \pm SD).

Of the 15 patients with MM, $4(27 \%)$ had migraine with aura and $11(73 \%)$ had migraine without aura (Table 1). There was no statistically significant difference between the 2 subgroups in the duration or timing of MM attack. All the patients had only one migraine attack during menstruation. The headache was unilateral in 6 cases $(40 \%)$ and bilateral in 9 cases $(60 \%)$.

Table 1 Brain hypoperfusion in 15 patients with menstrual migraine (MM), as revealed by SPECT during ovulation (midcycle) and during an MM attack

\begin{tabular}{clllll}
\hline Patient & Migraine & Localisation of the pain area & Laterality & & Hypoperfusion \\
\cline { 5 - 6 } & & & & Midcycle & During MM attack \\
\hline 1 & MWOA & Right orbitotemporal and vertex areas & Unilateral & Right parietal lobe & Right parietal lobe \\
2 & MWA & Right orbitotemporal area & Unilateral & - & Right occipital lobe and basal ganglia \\
3 & MWOA & Temporoparietal and vertex areas & Bilateral & - & - \\
4 & MWOA & Neck-occipital area & Bilateral & Right temporal lobe & - \\
5 & MWOA & Vertex and temporal areas & Bilateral & Right parietal lobe & - \\
6 & MWOA & Forehead-vertex area & Bilateral & - & - \\
7 & MWOA & Orbitotemporal area & Bilateral & - & - \\
8 & MWOA & Neck-occipital area & Bilateral & Right parietal lobe & - \\
9 & MWOA & Right vertex-occipital area & Unilateral & Left parietal lobe & Left parietal lobe \\
10 & MWA & Right orbitotemporal area & Unilateral & - & Right occipital lobe \\
11 & MWA & Right orbitofrontal area & Unilateral & - & Right temporal lobe \\
12 & MWOA & Left frontotemporal area & Unilateral & Left parietal lobe & Left temporal lobe \\
13 & MWOA & Neck-occipital-vertex area & Bilateral & Right parietal lobe & - \\
14 & MWA & Frontotemporoparietal area & Bilateral & - & Left parietal lobe \\
15 & MWOA & Temporoparieto-occipital area & Bilateral & - & -
\end{tabular}

MWOA, migraine without aura; $M W A$, migraine with aura 
Table 2 Perfusion activity in brain regions as assessed by SPECT. Values are the number of radioactively uptaken cells

\begin{tabular}{|c|c|c|c|c|c|c|c|c|c|c|c|c|}
\hline \multirow[t]{2}{*}{ Patient } & \multicolumn{6}{|c|}{ Midcycle } & \multicolumn{6}{|c|}{ MM attack } \\
\hline & $\mathrm{AF}$ & $\mathrm{PF}$ & $\mathrm{T}$ & $\mathrm{P}$ & MO & LO & $\mathrm{AF}$ & $\mathrm{PF}$ & $\mathrm{T}$ & $\mathrm{P}$ & MO & LO \\
\hline 1 & 0.94 & 1.12 & 0.93 & 1.11 & 1.14 & 1.11 & 1.09 & 1.12 & 0.90 & 1.11 & 1.15 & 1.16 \\
\hline 2 & 0.93 & 0.90 & 0.74 & 0.90 & 0.89 & 0.93 & 0.69 & 1.01 & 0.89 & 1.03 & 0.99 & 0.99 \\
\hline 3 & 1.20 & 1.10 & 0.89 & 1.08 & 1.15 & 1.13 & 2.55 & 1.45 & 0.81 & 1.05 & 1.43 & 1.48 \\
\hline 4 & 0.69 & 1.07 & 0.87 & 1.05 & 1.09 & 1.09 & 0.76 & 1.06 & 0.90 & 1.08 & 1.08 & 1.09 \\
\hline 5 & 0.92 & 0.93 & 0.84 & 0.94 & 0.95 & 0.93 & 0.98 & 1.01 & 0.90 & 1.02 & 1.04 & 1.03 \\
\hline 6 & 1.12 & 1.10 & 0.92 & 1.05 & 1.06 & 1.06 & 1.10 & 1.09 & 0.91 & 1.07 & 1.08 & 1.05 \\
\hline 7 & 1.74 & 0.91 & 0.69 & 0.88 & 0.97 & 1.32 & 0.57 & 1.01 & 0.85 & 1.02 & 0.10 & 1.00 \\
\hline 8 & 1.04 & 0.99 & 0.84 & 1.15 & 1.20 & 1.18 & 1.28 & 0.91 & 0.69 & 0.88 & 1.11 & 2.09 \\
\hline 9 & 0.85 & 1.18 & 0.99 & 1.15 & 1.20 & 1.18 & 1.28 & 0.91 & 0.69 & 0.88 & 1.11 & 2.09 \\
\hline 10 & 1.03 & 1.08 & 0.93 & 1.07 & 1.11 & 1.09 & 0.45 & 1.11 & 0.90 & 0.04 & 0.89 & 0.94 \\
\hline 11 & 1.38 & 1.06 & 0.88 & 1.09 & 1.11 & 1.07 & 0.79 & 1.01 & 0.89 & 1.02 & 1.04 & 1.03 \\
\hline 12 & 4.16 & 1.04 & 1.02 & 0.75 & 1.17 & 1.53 & 2.33 & 1.18 & 0.97 & 1.20 & 1.22 & 1.18 \\
\hline 13 & 0.93 & 1.05 & 0.91 & 1.05 & 1.10 & 1.10 & 0.47 & 1.04 & 0.98 & 1.10 & 1.00 & 1.05 \\
\hline 14 & 0.36 & 1.14 & 0.97 & 1.19 & 1.11 & 0.91 & 1.02 & 0.99 & 0.84 & 0.97 & 1.02 & 1.03 \\
\hline 15 & 0.55 & 1.03 & 0.93 & 1.07 & 0.95 & 0.97 & 0.79 & 1.04 & 0.88 & 1.06 & 1.07 & 1.03 \\
\hline
\end{tabular}

$A F$, anterior frontal lobe; $P F$, posterior frontal lobe; $T$, temporal lobe; $P$, parietal lobe; $M O$, mid-occipital lobe; $L O$, lateral occipital lobe

Brain SPECT was performed on day 1 or 2 of the MM attack (mean \pm SD, $1.5 \pm 0.5$ ). None of the patients reported headache lasting less than 1 day.

In the visual evaluation, we observed hypoperfusion in 7 of the 15 patients during MM attack (Table 1). Four of these 7 patients, specifically the 4 patients with migraine with aura, had normal perfusion in the middle of the cycle. The hypoperfusion areas revealed during the MM attack in these patients were the right occipital lobe two patients, in the right temporal lobe in one patient and the left parietal lobe in the remaining patient. Hypoperfusion was observed both during the MM attack and in the middle of the menstrual cycle, in 3 of the 15 patients (Table 1). Hypoperfusion was observed in 4 other patients at midcycle but not during the MM attack, and the final 4 patients had no evidence of brain hypoperfusion

We quantitatively compared the perfusions of the frontal lobes, temporal lobes, parietal lobes and occipital lobes during the MM attack and in the middle of the menstrual cycle with Ceretec programme. There was no statistically significant difference in perfusion activities between the two monitoring periods for any patient $(p>0.05)$ (Table 2). Putting the data together, independently from the pattern of each patient, we also compared cerebral blood flow in midcycle and MM attack, but the difference did not reach the level of statistical difference for patients with migraine without aura (data not shown). No comparison was possible for MWA patients due to the small number of patients.

\section{Discussion}

The pathogenesis of migraine has not yet been defined clearly. Neurogenic, vascular and humoral factors are putative mechanisms involved [8-11].

In particular, two theories have been proposed to explain migraine pathogenesis and the debate still continues. The first is the transient cerebral arterial constriction that causes reduced brain perfusion and migraine aura while the subsequent headache results from the intra- and/or extracranial arterial vasodilatation. The second one is cortical spreading depression, which induces an oligemia originating in the posterior brain and progressing anteriorly, and vascular abnormalities are considered only secondary phenomena [11, 12].

The pathogenetic mechanisms underlying mestruationassociated migraine attacks are scarcely known. Estrogen withdrawal can postpone the migraine [13-16]. One report suggested that MM attacks could be prevented by suppressing the progesterone-estrogen fluctuation in menstrual cycle [17]. Other authors, on the contrary, have not supported the hormonal theory because hormonal therapy is not beneficial to every patient suffering from MM and sometimes it worsens the attacks in terms of increased frequency and intensity $[13,18]$. Additional studies stated that not only estrogen and progesterone but also prostaglandin and magnesium deficiency took part in MM pathogenetic mechanisms $[13,15,18]$. 
Other abnormalities have been shown in menstrual migraine patients such as changes in the level of prolactin, dysregulation of opioids, disordered sympathetic activity, impaired carbohydrate tolerance, platelet dysfunction, vitamin deficiencies and inherited immune pattern [19]. Neurogenic mechanisms have also been hypothesized in MM attacks but few studies have been performed until now in this regard. Among them, a brainstem auditory evoked response (BAER) study revealed a neural abnormality which might be at brainstem level [16] and electroencephalographic findings showed a significant increase in mean alpha amplitude, theta activity and mild paroxysms, particularly in premenstrual syndrome [17].

It has been known for several years that vascular changes occur during migraine attacks but no studies have been carried out until now on mestruation-associated migraine attacks. Changes in $\mathrm{rCBF}$ were found in migraineurs, assessed in interictal periods [20-26].

On the other hand, hormonal variations (in particular estrogen levels) have been evidenced to induce an increase in the cerebral blood flow velocity and pulsatility indices [27]. Moreover, haemodynamic changes have been found at different phases in the menstrual cycle and also higher standard Doppler indices have been demonstrated in the luteal phase compared to the follicular phase in the middle cerebral artery (MCA) [28]. Increased ventilation and subsequent lowering of alveolar $\mathrm{CO}_{2}$ pressure, secondary to a raised progesterone level in mid-luteal phase, could account for the observed changes within the MCA [28]. Studies of cerebral perfusion during MM attack are lacking until now. In our study we found hypoperfusion during MM attack in 7 patients. Three of these patients had hypoperfusion also at midcycle. In 2 of these cases, hyper- fusion was observed in the same brain areas during MM attack as during midcycle.

On the other hand, hypoperfusion was evident in 3 patients at parietal and 1 at temporal area and only at midcycle in 4 patients. As far as the midcycle is concerned, we were unable to verify the occurrence of ovulation because no methods such as body basal temperature or urinary sticks were used. This limits the possibility of demonstrating a clear relation between midcycle cerebral haemodynamic changes with hormonal ovulation, and we cannot discriminate whether the changes observed are related to these physiological variations or are, on the contrary, peculiar of migraine patients.

The different pattern observed among patients at midcycle could be related to the occurrence or non-occurrence of ovulation but no conclusion of this can be made due to the lack of definite evidence.

In this regard it should be pointed out that variations of brain perfusion should be verified also in healthy subjects not suffering from headache. The changes in cerebral haemodynamics should be finally investigated in women with non-menstrual migraine examined at midcycle.

As far as the hypoperfusion is concerned (during menstrual migraine attack), both neuronal and vascular changes are already favoured by estrogen withdrawal. However, no explanation can be given for the cases in which these changes have not been observed.

Further studies investigating cerebral blood flow, also involving attacks not associated with mestruation, should be carried out in this regard. This should allow us to discriminate whether a different pattern of cerebral haemodynamic changes is evidenced in MM and non-MM. They should involve cerebral SPECT, Doppler ultrasound and MCA evaluation.

\section{References}

1. MacGregor EA, Chia H, Vohrah RC (1990) Migraine and menstruation: a pilot study. Cephalalgia 10:305-310

2. Welch KMA, Darnley D, Simkins RT (1984) The role of estrogen in migraine. Cephalalgia 4:225-236

3. Fettes I (1999) Migraine in the menopause. Neurology 53[Suppl 1]:29-33

4. Welch KMA (1997) Migraine and ovarian steroid hormones. Cephalalgia 17[Suppl 20]:12-16
5. Isler H, Olesen J (1993) Historical background and classification. In: Olesen J, Tfelt-Hansen P, Welch KMA (eds) The headaches. Raven, New York, pp 1-11

6. Granella F, Sances G, Pucci E, Nappi RE, Ghiotto N, Nappi G (2000) Migraine with aura and reproductive life events: a case control study. Cephalalgia 20(8):701-707

7. Granella F, Sances G, Zanferrari C (1993) Migraine without aura and reproductive life events. A clinical epidemiological study in 1300 women. Headache 33:385-389
8. MacGregor EA (1996) Menstrual migraine: towards a definition. Cephalalgia 16:11-21

9. Carol AJ (1999) Management of menstrual migraine. Neurology 53[Suppl 1]:14-18

10. Edvinsson L, Jansen I (1993) Vasomotor responses and receptor characteristics of human cranial arteries and veins. In: Olesen J, TfeltHansen P, Welch KMA (eds) The headaches. Raven, New York, pp 117-127 
11. Ferrari MD (1993) Systemic biochemistry. In: Olesen J, Tfelt-Hansen P, Welch KMA (eds) The headaches. Raven, New York, pp 179-191

12. Maini CL, Turco GL, Castellano G, Liboni W, Padio V, Chianale G, Cornaglia G (1990) Cerebral blood flow and volume in symptom-free migraineurs: a SPECT study. Nucl Med 29:210-214

13. MacGregor EA, Chia H, Vohrah RC, Wilkinson M (1990) Migraine and menstruation: a pilot study. Cephalalgia 10(6):305-310

14. Somerwille BW (1971) The role of progesterone in menstrual migraine. Neurology 21:853-859

15. Pitcher DW, Papouchado M, Channer K, James MA (1996) Prevention of menstrual migraine by percutaneous oestradiol. BMJ 293:1540

16. Howard R, Mason P, Taghavi E (1992) Brainstem suditory evoked responses during the menstrual cycle in women with and without premenstrual syndrome. Biol Psychiatry 32:682-690

17. Leary PM, Batho K (1979) Changes in the EEG related to the menstrual migraine. S Afr Med J 55:666-668
18. Silberstein S, Merriam G (1991)

Estrogens and progestins and headache. Neurology 41:86-93

19. MacGregor A (1999) Migraine in women. Martin Dunitz, London, pp 33-46

20. Lauritzen M, Olesen J (1984) Regional cerebral blood flow during migraine attacks by xenon-133 inhalation and emission tomography. Brain 107:447-461

21. Podreka I, Suess E, Goldenberg G, Steiner M, Brucke T, Muller C et al (1987) Initial experience with technetium-99m-HMPAO brain SPECT. J Nucl Med 28:1657-1666

22. Levine SR, Welch KMA, Ewing JR, Robertson WM (1987) Asymmetric cerebral blood flow patterns in migraine. Cephalalgia 7:245-248

23. Friberg I, Olesen J, Iversen H, Nicolic I, Sperling B, Lassen NA et al (1994) Interictal "patchy" regional cerebral blood flow patterns in migraine patients. A single photon emission computerized tomographic study. Eur J Neurol 1:35-43
24. Facco E, Munari M, Baratto F, Behr AU, Dal Palu A, Cesaro S et al (1996) Regional cerebral blood flow (rCBF) in migraine during the interictal period: different $\mathrm{rCBF}$ patterns in patients with and without aura. Cephalalgia 16:161-168

25. Soriani S, Feggi L, Battistella PA, Arnaldi C, De Carlo I, Stipa S (1997) Interictal and ictal phase study with Tc 99m HMPAO brain SPECT in juvenile migraine with aura. Headache 37:31-36

26. De Benedittis G, Ferrari Da Passano C, Granata G, Lorenzetti A (1999) CBF changes during headache-free periods and spontaneous/induced attacks in migraine with and without aura: a TCD and SPECT comparison study. J Neurosurg Sci 43:141-146

27. Shamma FN, Fayad P, Brass L, Sarrel P (1992) Middle cerebral artery blood velocity during controlled ovarian hyperstimulation. Fertil Steril 57(5):1022-1025

28. Brackley KJ, Ramsay MM, Pipkin FB, Rubin PC (1999) The effect of the menstrual cycle on human cerebral blood flow: studies using Doppler ultrasound. Ultrasound Obstet Gynecol 14(1):52-57 